\title{
Endocarditis Marántica asociada a linfoma de Hodgkin
}

\author{
Diego Arnaldo Medina Acuña ${ }^{1}$, Lorena Gabriaguez Gonzalez ${ }^{2}$, Melissa Raquel Vargas Viveros ${ }^{3}$, $*$ Carlos \\ Miguel Rios-González ${ }^{4}$ \\ ${ }^{1}$ Universidad Católica Nuestra Señora de la Asunción. Facultad de Medicina. Postgrado en Medicina Interna. \\ Asunción, Paraguay \\ ${ }^{2}$ Hospital Central del Instituto de Previsión Social. Servicio de Clínica Médica. Asunción, Paraguay \\ ${ }^{3}$ Universidad Nacional de Itapúa. Facultad de Medicina. Encarnación, Paraguay \\ ${ }^{4}$ Universidad Nacional de Caaguazu, Facultad de Ciencias Médicas, Cnel. Oviedo, Paraguay
}

\section{Cómo referenciar este artículo/ How to reference this article:}

\begin{abstract}
Medina Acuña DA, Gabriaguez González L, Vargas Viveros, MR, Ríos-González CM. Asociación de la dilatación auricular izquierda con los trastornos del sistema de conducción en pacientes con hipertensión arterial sistémica. Mem. Inst. Investig. Cienc. Salud. 2019; 17(3): 72-75
\end{abstract}

\section{RES U ME N}

La endocarditis trombótica no bacteriana (ETNB) se define como la presencia de trombos estériles sobre las válvulas cardiacas, corresponden a agregados de plaquetas y fibrina no infectados asociados a estados de hipercoagulabilidad como las neoplasias malignas, en donde se denomina también endocarditis marántica. Se presenta el caso de un paciente con historia de síndrome febril prolongado al que se le inició tratamiento antibiótico por una endocarditis infecciosa (EI) por presentar una imagen compatible con vegetación en el ecocardiograma transtorácico. Sin embargo, el paciente persistió febril y sin mejoría clínica, hasta que la biopsia de un ganglio cervical diagnosticó un linfoma de Hodgkin mejorando posteriormente con el tratamiento hemato específico.

Palabras clave: Endocarditis marántica, hipercoagulabilidad, neoplasia, linfoma.

\section{Marantic Endocarditis associated with Hodgkin's Lymphoma}

\section{A B S T R A C T}

Non-bacterial thrombotic endocarditis (NBTE) is defined as the presence of sterile thrombi on the heart valves, correspond to non-infected platelet and fibrin aggregates associated with hypercoagulable states such as malignant neoplasms, which is also called Marantic Endocarditis. We present the case of a patient with a history of prolonged febrile syndrome who underwent antibiotic treatment for an Infective Endocarditis (EI) due to a compatible image with vegetation in the transthoracic echocardiogram. However, the patient persisted feverish and without clinical improvement, until the biopsy of a cervical ganglion diagnosed a Hodgkin lymphoma, improving later with the specific hemato treatment.

Keywords: marantic endocarditis, hipercoagulability, neoplasia, limphoma.

\section{INTRODUCCIÓN}

La endocarditis trombótica no bacteriana (ETNB) fue descrita por primera vez en el año 1888 por Ziegler. Desde entonces ha recibido diversas denominaciones tales como endocarditis marántica (cuando se presenta en neoplasias) o endocarditis de Libman Sacks (cuando se presenta en enfermedades reumatológicas como el lupus eritematoso sistémico). En todos los casos corresponde a un trombo no infectado, formado por plaquetas y fibrinas, que asienta sobre el endocardio valvular y asociado a estados de hipercoagulabilidad ${ }^{(1)}$.

Fue el médico francés Armand Trousseau en $1865^{(2)}$ quien describió por primera vez la asociación entre cáncer e hipercoagulabilidad. En la fecha, numerosas son las situaciones

* Los autores no declaran conflicto de interés

Fecha de recepción: julio 2019. Fecha de aceptación: agosto 2019

*Autor correspondiente: Carlos Miguel Ríos González. Universidad Nacional de Caaguazu, Facultad de Ciencias Médicas, Cnel. Oviedo, Paraguay

Email: carlosmigue_rios@live.com 
patológicas que generan un estado procoagulante, pudiendo aparecer en pacientes con neoplasias malignas, enfermedades mieloproliferativas, como complicación de una sepsis, en quemaduras graves, en pacientes con síndrome antifosfolípido primario, en el lupus eritematoso sistémico, en el síndrome de inmunodeficiencia adquirida ${ }^{(3)}$ y en enfermedades pulmonares que cursan con hipoxia ${ }^{(4)}$.

En el Paraguay, no se encuentra ningún caso similar, constituyendo de esta manera el primer caso reportado, por lo que a continuación, presentamos un caso de endocarditis marántica asociado a un proceso linfoproliferativo.

\section{PRESENTACION DEL CASO}

Paciente masculino, 42 años de edad, sin patologías de base conocidas. Acude por historia de síndrome febril prolongado, graduada hasta $38,5^{\circ}$, sin predominio horario, que cedía en crisis con sudoración profusa. Se acompaña de astenia y pérdida del apetito con disminución de $7 \mathrm{~kg}$ de peso.

Al examen físico no se encontraron datos patológicos de valor, paciente lúcido, ubicado, colaborador con el interrogatorio. Al examen cardiaco no se auscultaba soplos, el ritmo era regular y sinusal por electrocardiograma.

En la analítica sanguínea llamaba la atención PCR aumentado, anemia moderada (normocítica/normocrómica), leucocitos en rango pero con neutropenia leve y LDH aumentada. Orina simple no patológica. (Tabla 1).

Tabla 1: Analítica sanguínea del paciente a su ingreso.

\begin{tabular}{lll}
\hline Hemograma & & Perfil renal \\
\hline Glob. Blancos & 4.900 & Urea:35 \\
$\mathrm{N}$ & $27 \%$ & Creat: 0.85 \\
$\mathrm{~L}$ & $46 \%$ & Electrolitos \\
Hemoglobina & 9,1 & Potasio: 3,8 \\
VCM / CHCM & $85 / 32$ & Sodio: 138 \\
Plaquetas & 140.000 & LDH \\
PCR / VSG & $104 / 61$ & 894 \\
\hline
\end{tabular}

Por síndrome febril prolongado se solicitó serología viral completa para VIH, VDRL, hepatitis viral, toxoplasmosis, citomegalovirus, rubéola, entre otros, todos retornaron normales o dentro de rango. Se realiza hemocultivo $x 2$ que retorna sin desarrollo bacteriano. Ecografía abdominal informa esplenomegalia leve, no palpable al examen físico.

Ecocardiografía transTorácica (ETT) encuentra imagen sugerente de vegetación sobre válvula mitral. En ese contexto se inicia tratamiento antibiótico empírico para endocarditis infecciosa (EI) con ampicilina, oxacilina y gentamicina, previa toma de hemocultivos por 6 muestras. La imagen compatible con vegetación se confirma por ecocardiografía transesofágica (ETE), que encuentra imagen de $9 \times 6 \mathrm{~mm}$ en la válvula mitral (Figura 1). 


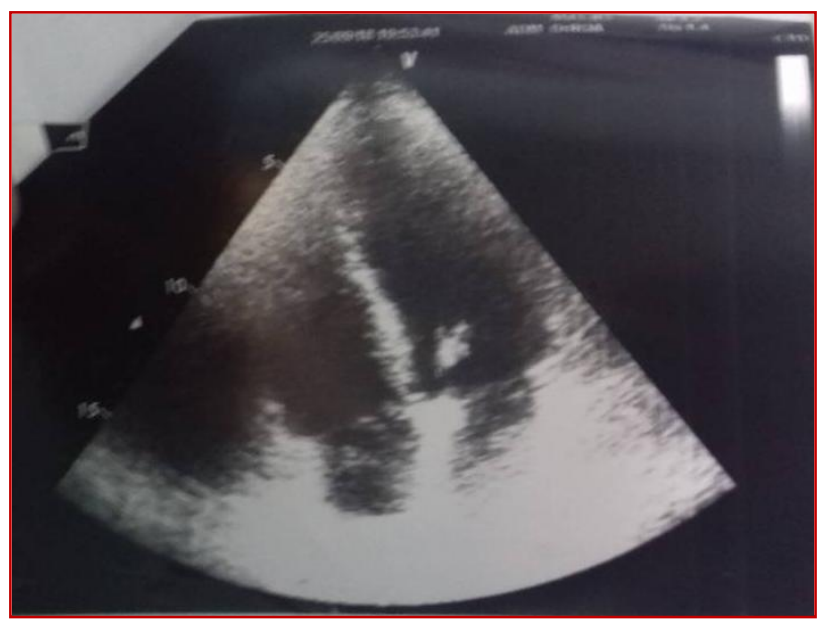

Figura 1: Imagen de 9x6mm sobre la válvula mitral observado por ETE

A pesar del tratamiento antibiótico instaurado el paciente no presentó mejoría, continuó presentando picos febriles de hasta $38,5^{\circ}$ en forma diaria, acompañados de sudoración profusa. Al día 7 los hemocultivos persisten negativos, ante sospecha de EI por gérmenes de cultivo difícil, se rota antibióticos a Ceftriaxona + Vancomicina. Se solicita serología para brucelosis, Bartonella, Coxiella burnetii que retornan negativos. Además, se solicita dosaje de procalcitonina que retorna bajo. Por persistencia de la fiebre, se realiza barrido tomográfico con doble contraste que informa esplenomegalia y múltiples adenomegalias axilares, mediastinales y retroperitoneales. En paralelo, el paciente presentaba disminución paulatina de leucocitos, hemoglobina y plaquetas en el hemograma. Ante sospecha de proceso linfoproliferativo, se consigue biopsiar un ganglio axilar, cuyo estudio anatomopatológico informa linfoma de Hodgkin, variedad mixta. El paciente inicia en forma inmediata tratamiento hemato específico con quimioterapia y metilprednisolona, a partir del cual presenta gran mejoría clínica y resolución de la fiebre hasta el día del alta.

Por la evolución clínica que presentó el paciente, los hemocultivos negativos, la procalcitonina baja, las pruebas serológicas negativas y la falta de respuesta a los antibióticos administrados, sumados al estado de hipercoagulabilidad del linfoma, se cataloga el caso como una endocarditis trombótica no bacteriana o endocarditis marántica. Se suspenden los antibióticos, el paciente continuó quimioterapia y en ETE realizada al mes, ya no observó imagen compatible con vegetación sobre la válvula mitral.

\section{DISCUSIÓN}

La ETNB es una entidad caracterizada por la presencia de vegetaciones estériles, constituidas por acúmulos de fibrina y plaquetas. Este proceso ha sido descrito en numerosas situaciones clínicas, siendo las más frecuentes las enfermedades neoplásicas sólidas, sobre todo en su fase terminal ${ }^{(1)}$. Situación que contrasta con nuestro paciente, cuyo hallazgo precedió al diagnóstico y estuvo asociado a una neoplasia hematológica. Son escasos los reportes de endocarditis marántica asociados a procesos linfoproliferativos.

La fisiopatología no se conoce en forma exacta, pero la mayoría de las literaturas consultadas coinciden en que exista un daño previo del endotelio, por la secreción del factor de necrosis tumoral y/o interleucinas 1 en los pacientes oncológicos, sumados al estado de hipercoagulabilidad ${ }^{(5)}$.

Encontramos datos de que el $15 \%$ de los pacientes con cáncer presentará un evento tromboembólico durante su curso clínico. Tales como; tromboembolismo venoso, tromboflebitis superficial migratoria, trombosis arterial, coagulación intravascular diseminada, microangiopatía trombótica y, rara vez, endocarditis trombótica no bacteriana, también conocida como endocarditis marántica ${ }^{(6,7)}$._ El $80 \%$ de los casos de endocarditis marántica están asociados con una enfermedad maligna subyacente ${ }^{(8)}$.

La asociación neoplásica más frecuente es con el adenocarcinoma, según describe la literatura científica ${ }^{(2-5,9)}$. La válvula mitral es la afectada con más frecuencia, seguida de la 
aórtica $^{(5,10)}$. Nos llama la atención, la falta de asociación o los escasos reportes de ETNB con neoplasias hematológicas, a pesar de que se narran en las literaturas médicas ${ }^{(4,8,10)}$.

La ETNB suele ser silenciosa clínicamente, cuando se manifiesta se caracteriza por soplos, arritmias cardiacas o tromboembolismo sistémico que es la complicación más grave $^{(7)}$.

El diagnóstico de ETNB es difícil, y debe reunir una serie de criterios. Los más descritos en la literatura son los criterios de Duke. La ETT es un método muy útil, pero resulta poco sensible para las vegetaciones de pequeño tamaño. Por lo que, cuando la sospecha clínica es alta, la ETE es fundamental para confirmar la presencia de vegetación sobre las válvulas. Es importante destacar que las lesiones valvulares de la ETNB resultan ecocardiográficamente indistinguibles de las observadas en la EI, por lo que es imperativo establecer un diagnóstico diferencial con esta entidad ${ }^{(10)}$. Este diagnóstico es especialmente dificultoso con los casos de EI con hemocultivos negativos, debiendo sospecharse un origen infeccioso hasta que el contexto clínico y la evolución del paciente demuestren lo contrario. En nuestro paciente, la persistencia de la fiebre a pesar de los antibióticos indicados, los resultados negativos de los hemocultivos, el dosaje bajo de procalcitonina y los resultados negativos de todas las pruebas serológicas realizadas para excluir la presencia de otros microorganismos causantes de EI, apoyaron el diagnóstico de ETNB o endocarditis marántica asociada a linfoma de Hodgkin.

En cuanto al tratamiento, es fundamental el control de la enfermedad de base. Muchos artículos describen el uso de heparina intravenosa para prevenir los fenómenos tromboembólicos ${ }^{(1)}$. Mientras que la anticoagulación a largo plazo solo está indicada en pacientes con síndrome antifosfolípido primario 0 en aquellos con episodios de tromboembolización ${ }^{(10)}$. No obstante, no existen datos concluyentes que aconsejen un tratamiento definitivo. Nuestro paciente recibió HBPM a dosis profilácticas durante su tiempo de internación, sin anticoagulación a largo plazo al alta. Con el tratamiento quimioterápico, el trombo se resolvió en la ETE de control realizada al mes.

Tras una exhaustiva revisión de la literatura mundial, son numerosos los casos de endocarditis marántica asociada a tumores sólidos, pero solo encontramos un caso reportado asociado a una neoplasia hematológica. Por lo cual, según nuestro conocimiento, este corresponde al primer caso de endocarditis marántica asociado a linfoma de Hodgkin, no solo para el país sino también para la literatura regional.

\section{REFERENCIAS BIBLIOGRAFÍCAS}

1. López JA, Ross RS, Fishbein MC, Siegel RJ. Nonbacterial thrombotic endocarditis: A review. Am Heart J 1987; 113: 773-84.

2. Trousseau A. Clinique Médicale de I'HôtelDieu de Paris. Paris: Ballière; Phlegmasia alba dolens; 1865: 654-712.

3. Kaul S, Fishbeim MC, Siegel RJ. Cardiac manifestations of acquierd inmunodeficiency syndrome: a 1991 update. Am Heart J 1991; 122: 535-44.

4. Truskinovsky AM, Hutchins GM. Association between nonbacterial thrombotic endocarditis and hypoxigenic pulmonary diseases. Virchows Arch 2001; 438: 35761.

5. Vilacosta I, San Román JA, Sarriá C. Endocarditis trombótica no bacteriana. En: Vilacosta I, Sarriá C, San Román JA, editores. Endocarditis infecciosa. Barcelona: Prous Science, 2002. Falta paginas.

6. Khalil J, Bensaid B, Elkacemi H, Afif $M$, Bensaid $Y$, Kebdani T. Venous thromboembolism in cancer patients: an underestimated major health problem. World J Surg Oncol 2015; 13: 204. doi: 10.1186/s12957-015-0592-8.

7. Lobo Ferreira T, Alves R, Judas T, Delerue MF. Marantic endocarditis and paraneoplastic pulmonary embolism. BMJ Case Rep. 2017; 2017. pii: bcr-2017220217. doi: $10.1136 /$ bcr-2017-220217.

8. Ali M. Non-bacterial thrombotic endocarditis and subclinical myopericarditis in a patient with advanced rectal cancer. BMJ Case Rep. 2015; 2015. doi: $10.1136 /$ bcr-2015-212820

9. Ludwig $M$, Deppisch MD, Olusegun MD. Non-bacterial thrombotic endocarditis. Clinicopathologic correlations. Am Heart J. 1976;92:723-9.

10. Reisner SA, Brenner B, Haim N, Edoute $Y$, Markiewick W. Echocardiography in nonbacterial thrombotic endocarditis: from autopsy to clinical entity. Am Soc Echocardiogr 2000; 13: 876-81. 